\title{
Dynamique des sédiments fins dans une zone côtière à forte turbidité à proximité de l'embouchure de la Loire
}

\author{
Martin SANCHEZ ${ }^{1}$ \\ ${ }^{1}$ Université de Nantes, UMR CNRS 6112 Planétologie et Géodynamique, \\ 2 rue de la Houssinière, 44322 Nantes, France. \\ martin.sanchez@univ-nantes.fr
}

\section{Résumé :}

La répartition des dépôts de sédiments fins dans un domaine côtier à proximité de l'estuaire de la Loire est présentée. Les courants de marée et la houle dans le domaine étudié sont décrits grâce à la mise au point d'une modélisation numérique 2DH. Une méthode basée sur l'étude des forces hydrodynamiques et de leur action sur les sédiments fins, est développée afin de cerner les tendances sédimentaires observées dans différents points du domaine étudié. L'application de cette méthode dans une région centrée sur la baie de Bourgneuf permet d'expliquer la forte accumulation de sédiments fins et les importants remaniements de sédiments observés dans cette baie.

\begin{abstract}
:
The distribution of fine sediment deposits in a coastal field near the estuary of the River Loire is presented. Tidal currents and swell heights are described by a numerical 2DH model implemented in

this study. A method, based on the study of hydrodynamic forces and their action on fine sediment dynamics, is developed in order to determine the sedimentary tendencies observed in several points of the studied field. The application of this method in an area centred on Bourgneuf bay makes it possible to explain the strong accumulation of fine sediments in this bay and the frequently observed high turbidity produced by resuspension.
\end{abstract}

\section{Mots-clés :}

Sédiment fin - Courant de marée - Houle - Erosion - Transport en suspension Dépôt - Envasement - Turbidité - Baie de Bourgneuf - Loire 


\section{Introduction}

Une accumulation de sédiments organiques fins du type vase est souvent observée dans les estuaires. L'envasement de ces sites est en relation avec l'intrusion saline (IPPEN, 1966) et avec les courants résiduels 2DV convergeant au fond vers un point dit nodal (ALLEN, 1972). Dans ces sites, la remise en suspension est à l'origine de la formation d'une masse d'eau à forte turbidité connue sous le nom de "bouchon vaseux". Ensuite, quand les eaux redeviennent calmes, les sédiments forment des dépôts de "crème de vase" pouvant conserver presque indéfiniment une structure peu dense (SANCHEZ \& LEVACHER, 2007).

Certaines régions côtières abritées de la houle sont aussi le siège d'importantes accumulations de vase. Les courants de marée résiduels 2DH ne semblent pas influer sur la localisation de ces accumulations.

Le but de cette note est de mettre en place une méthode rationnelle des tendances sédimentaires afin d'expliquer les accumulations des sédiments fins dans certaines régions côtières, autres que les estuaires. Cette méthode est basée sur l'étude des forces hydrodynamiques et de leur rôle sur la remise en suspension, le transport en suspension et le dépôt des matières en suspension.

\section{Site d'étude}

Cette étude concerne les côtes françaises de la façade atlantique à proximité de l'embouchure de la Loire. Les sédiments déposés dans cette région ont été étudiés par plusieurs auteurs (GOULEAU, 1975; VANNEY, 1977; LCHF, 1987 ; LESUEUR et al., 2001). Cinq principales zones d'accumulation de sédiments fins (fraction minérale inférieure à $63 \mu \mathrm{m}$ ) sont répertoriées (figure 1) :

a) L'estuaire de la Loire. Il est en relation directe avec les apports en suspension $\mathrm{du}$ fleuve qui représentent en moyenne $1.5 \times 10^{9} \mathrm{~kg} \mathrm{an}{ }^{-1}$. En morte-eau, les accumulations de "crème de vase" peuvent atteindre une masse sèche de $1 \times 10^{9} \mathrm{~kg}$.

b) La zone de la Lambarde située au nord-ouest de l'île de Noirmoutier. L'envasement de ce site résulte de l'évacuation des vases de la Loire pendant les crues. Un envasement lié au déversement par clapage des sédiments dragués dans le chenal de navigation de la Loire est aussi à prendre en compte.

c) La baie de Vilaine. Elle est presque complètement envasée du fait d'une forte atténuation de la houle dans ce secteur. Un très important stock de "crème de vase" est également présent dans cette zone.

d) La Grande Vasière. Elle est située à environ $80 \mathrm{~km}$ du littoral dans une zone délimitée approximativement par les isobathes $-100 \mathrm{~m}$ et $-120 \mathrm{~m}$ (par rapport au zéro hydrographique). Les sédiments sont un mélange de sable hétérogène avec une fraction fine représentant moins de $25 \%$ des matières. Il s'agirait d'un dépôt fossile (BARUSSEAU, 1973) épais de quelques dizaines de centimètres. 


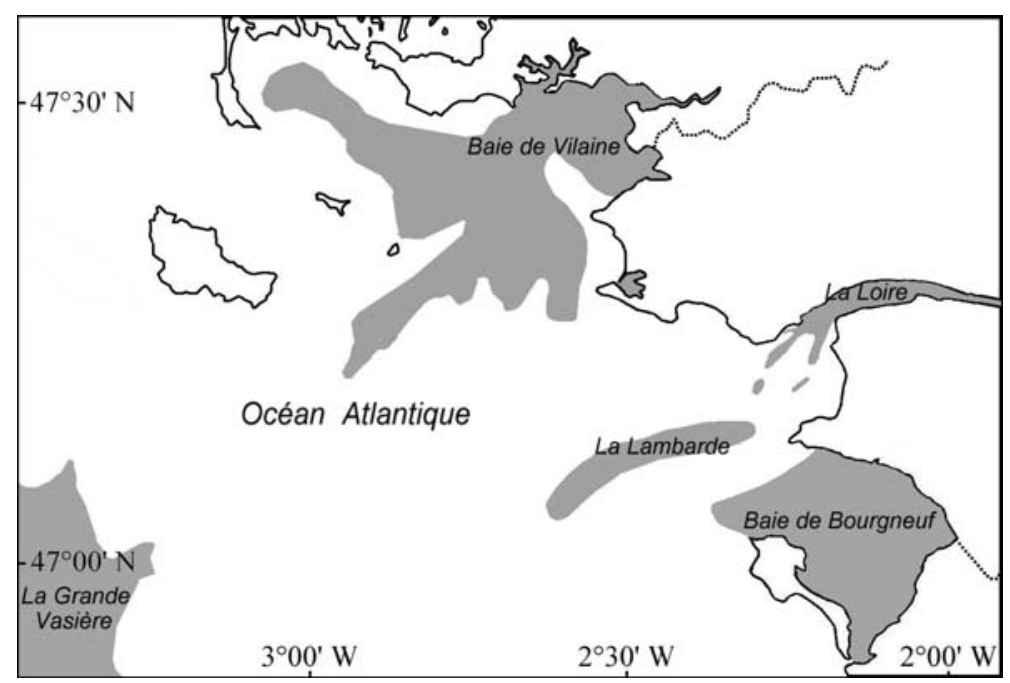

Figure 1. Dépôts marins de sédiments fins à proximité de l'embouchure de la Loire. Carte élaborée à partir de données publiées par GOULEAU (1975), VANNEY (1977), ALLEN \& CASTAING (1977) et LCHF (1987).

e) La baie de Bourgneuf située juste au sud de l'estuaire externe de la Loire. Cette baie est une dépression tectonique partiellement séparée de l'océan par l'île de Noirmoutier et, par conséquent bien abritée par rapport à la houle. Les vases couvrent la plus grande partie de la baie à l'exception de la partie haute des estrans où les sables prédominent.

Cette étude est centrée d'une façon particulière sur la baie de Bourgneuf. Une couche de 5 à $7 \mathrm{~m}$ de sédiments déposés à la fin de la transgression flandrienne couvre presque la totalité de cette baie (DELANOË et al., 1971) sauf quelques affleurements rocheux. Les sédiments fins sont présents dans des proportions très variables comprises entre 1\% et plus de 50\% (GOULEAU, 1975). Une importante accumulation de "crème de vase" a été observée couvrant les fonds sur une surface de $2 \mathrm{~km}$ par $12 \mathrm{~km}$ dans la zone Est de la baie (GOULEAU, 1975).

Une zone de forte turbidité avec des concentrations dépassant les $0.2 \mathrm{~kg} \mathrm{~m}^{-3}$ est souvent décelée sur des images satellite à partir de la mesure de la réflectance de la surface océanique (FROIDEFOND et al., 2003). Cette zone turbide peut couvrir toute la baie de Bourgneuf et s'étaler vers l'ouest et le sud de l'île de Noirmoutier jusqu'à une dizaine de kilomètres de la côte.

En cas de tempête, la houle et les clapots peuvent remettre en suspension une grande quantité de sédiments fins qui se dispersent horizontalement sous l'effet des courants de marée. La pérennité des fortes turbidités dans cette zone ne peut alors s'expliquer que par l'existence d'un mécanisme ramenant vers leurs points de départ ces sédiments dispersés. Autrement, ils finiraient par être expulsés vers le large. 


\section{Méthodes}

\subsection{Les agents hydrodynamiques}

Trois types d'agents hydrodynamiques sont pris en compte : les courants de marée, la houle se propageant des grandes profondeurs vers la côte et la mer de vent ou clapot généré localement par le vent.

Le domaine maritime étudié est représenté sur la figure 2. Il comprend l'estuaire de la Loire et une bande large d'environ $100 \mathrm{~km}$ devant les côtes de la région des Pays de la Loire. La bathymétrie est donnée avec une densité moyenne d'un point au $\mathrm{km}^{2}$ sauf aux environs de la baie de Bourgneuf où cette densité est 10 fois supérieure. Dans l'estuaire de la Loire, les sections considérées sont rectangulaires respectant une condition d'équivalence des débits.

Les courants de marée sont évalués dans chaque point du domaine étudié grâce à la mise au point d'une modélisation numérique 2DH, effectuée à l'aide du logiciel Flow Model MIKE 21 (DHI, 2007a). Les valeurs instantanées des conditions de frontière océanique liées à la marée, sont calculées par le propre logiciel, par une méthode des constantes harmoniques, alors que les conditions de frontière fluviales ont été fixées pour reproduire un débit d'étiage de $100 \mathrm{~m}^{3} \mathrm{~s}^{-1}$. La marée prédite à Saint-Nazaire et un atlas de courants de marée (SHOM, 1990) ont permis l'ajustement du modèle. Les courants de marée sont filtrés a posteriori de façon à supprimer de ceux-ci les composantes résiduelles eulériennes.

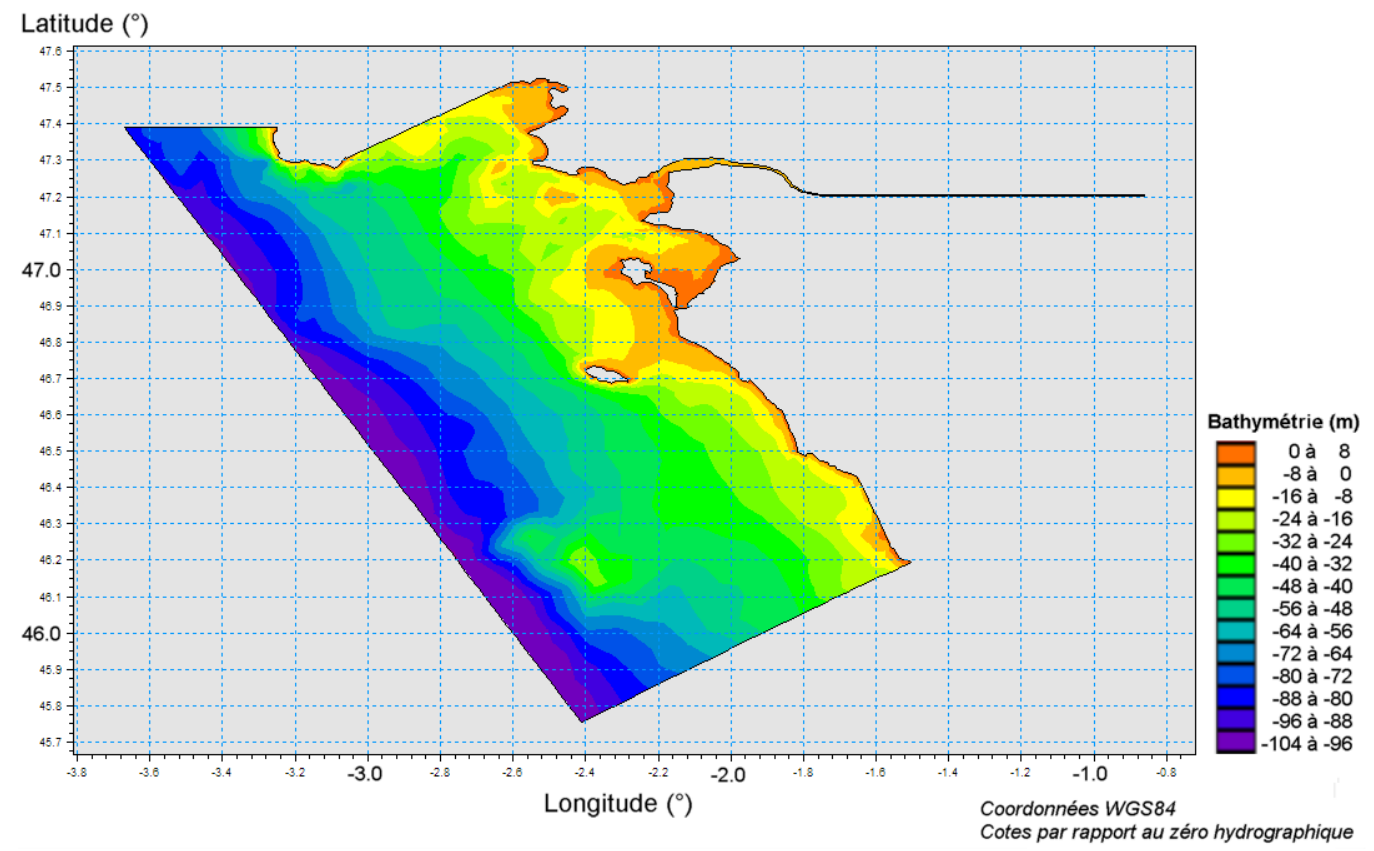

Figure 2. Représentation du domaine maritime étudié avec la bathymétrie. 
Un modèle de propagation de la houle est également mis en oeuvre à l'aide du logiciel Spectral Waves MIKE 21 (DHI, 2007b). Des corrélogrammes hauteurpériode issues de deux stations de mesure de la base de données CANDHIS (site web) du CETMEF sont utilisés pour étalonner des fonctions de transfert entre le large et les points d'étude. Ces deux stations sont les suivantes :

- Bouée Ile d'Yeu n ${ }^{\circ} 08502$ directionnelle $\left(02^{\circ} 25.65^{\prime} \mathrm{W}, 47^{\circ} 41.45^{\prime} \mathrm{N}\right)$.

- Bouée St-Nazaire $n^{\circ} 04401$ non directionnelle $\left(02^{\circ} 20.30^{\prime} \mathrm{W}, 47^{\circ} 09.20^{\prime} \mathrm{N}\right)$.

La valeur moyenne de la hauteur de houle significative a été évaluée en 36 points du domaine étudié à l'aide des fonctions de transfert étalonnées. Enfin, des statistiques du vent ont permis le calcul de la hauteur moyenne du clapot de mer de vent dans les points situés dans la baie de Bourgneuf, où la hauteur est limitée par le fetch.

\subsection{Caractérisation des forces hydrodynamiques}

Les forces hydrodynamiques agissant sur les sédiments sont caractérisées à travers la contrainte tangentielle au fond $\tau$. Dans cette étude, trois composantes de la contrainte $\tau$ sont considérées :

- Une contrainte quasi-stationnaire $\tau_{(1)}$ due au courant de marée.

- Une contrainte cyclique $\tau_{(2)}$ due à la propagation de la houle.

- Une contrainte cyclique $\tau_{(3)}$ due à la propagation de la mer de vent.

La contrainte $\tau_{(l)}$ est calculée avec la formulation de Strickler en fonction de la vitesse débitante. Pour $\tau_{(2)}$ et $\tau_{(3)}$, les calculs sont effectués en valeur de pointe, avec des formulations issues des théories des couches limites laminaire et turbulente (FREDSØE \& DEIGAARD, 1992). Ces calculs prennent en compte la vitesse orbitale horizontale près $\mathrm{du}$ fond, vitesse induite par la propagation des ondes de gravité océaniques.

Pour les études des tendances sédimentaires à moyen et long terme effectuées par la suite, il est admis que la marée, la houle et la mer de vent, sont trois processus non corrélés et donc indépendants. Une valeur probable de $\tau$ est ainsi calculée par l'application d'un théorème de Pythagore généralisé comme suit :

$$
\tau=\sqrt{\tau_{(1)}^{2}+\tau_{(2)}^{2}+\tau_{(3)}^{2}}
$$

\subsection{Description de la méthode rationnelle mise au point}

Une méthode est mise au point dans le but d'étudier les tendances qui résultent de l'action des agents hydrodynamiques sur des sédiments fins qui sont principalement transportés en suspension. Pour analyser ces tendances, la vitesse résultante $v_{r}$ est évaluée pour certaines particules sur un cycle de marée complet. 
Deux seuils de la contrainte tangentielle au fond $\tau^{e}$ et $\tau^{d}$ sont utilisés pour paramétrer la remise en suspension et le dépôt, respectivement $\left(\tau^{e}>\tau^{d}\right)$. Le passage par $\tau=\tau^{e}$ par valeurs croissantes de $\tau$, définit l'instant caractéristique de la remise en suspension et du début de transport des particules étudiées. Le passage par $\tau=\tau^{d}$ par valeurs décroissantes de $\tau$, définit l'instant caractéristique du dépôt de ces particules. Il doit être noté que le seuil $\tau^{e}$ n'est pas celui de la loi de début d'érosion de PARTHENIADES (1965) et que le seuil $\tau^{d}$ n'est pas non plus celui de la formulation de taux de dépôt de KRONE (1986).

Deux marées ont été examinés, à savoir, une vive-eau moyenne de coefficient 95 et une morte-eau moyenne de coefficient 45. Pour chaque marée, quatre conditions de houle de période $10 \mathrm{~s}$ sont considérées. Elles sont caractérisées par les valeurs de la hauteur significative $H_{o}$ au large de l'île d'Yeu. Ces valeurs sont :

- $H_{o}=0.5 \mathrm{~m}$, hauteur très faible.

- $H_{o}=1 \mathrm{~m}$, hauteur faible.

- $H_{o}=2 \mathrm{~m}$, hauteur moyenne (arithmétique).

- $H_{o}=4 \mathrm{~m}$, hauteur élevée.

Les désignations de hauteur de houle ci-dessus ne sont pas conformes à l'échelle usuelle de Douglas; il s'agit d'une échelle établie sur la base des valeurs observées au large de l'île d'Yeu. Enfin, il doit être précisé que la mer de vent est toujours représentée par des vagues d'une période de $2.4 \mathrm{~s}$ et d'une hauteur de $0.3 \mathrm{~m}$, et que le seuil $\tau^{e}$ est fixé au double de la valeur de $\tau^{d}$.

\section{4. $\quad$ Résultats}

Suivant les valeurs de la vitesse résultante $v_{r}$, et des contraintes maximale $\tau_{\max }$ et minimale $\tau_{\min }$ au cours d'un cycle de marée complet, plusieurs tendances sédimentaires sont déduites. Celles-ci sont synthétisées dans le tableau 1. Les figures 3 et 4 présentent les résultats relatifs aux tendances sédimentaires modélisées dans les 36 points étudiés pour des marées de vive-eau et de morteeau, respectivement.

Tableau 1. Synthèse des tendances sédimentaires modélisées.

\begin{tabular}{lll}
\hline Valeurs de $\tau$ & Vitesse résultante $v_{r}$ & Tendance sédimentaire déduite \\
\hline$\tau_{\min }<\tau^{d}$ et $\tau_{\max }<\tau^{d}$ & $\sim 0 \mathrm{~m} \mathrm{~s}^{-1}$ & Dépôt généralisé \\
$\tau_{\min }<\tau^{d}$ et $\tau^{d}<\tau_{\max }<\tau^{e}$ & $\sim 0 \mathrm{~m} \mathrm{~s}^{-1}$ & Dépôt intermittent \\
$\tau_{\min }<\tau^{d}$ et $\tau_{\max }>\tau^{e}$ & $\sim 0 \mathrm{~m} \mathrm{~s}^{-1}$ & Equilibre entre dépôt et érosion \\
$\tau_{\min }<\tau^{d}$ et $\tau_{\max }>\tau^{e}$ & $<0.02 \mathrm{~m} \mathrm{~s}^{-1}$ & Transport net modéré \\
$\tau_{\min }<\tau^{d}$ et $\tau_{\max }>\tau^{e}$ & $>0.02 \mathrm{~m} \mathrm{~s}^{-1}$ & Transport net marqué \\
$\tau^{d}<\tau_{\min }<\tau^{e}$ et $\tau_{\max }>\tau^{e}$ & $\sim 0 \mathrm{~m} \mathrm{~s}^{-1}$ & Erosion intermittente \\
$\tau_{\min }>\tau^{e}$ et $\tau_{\max }>\tau^{e}$ & $\sim 0 \mathrm{~m} \mathrm{~s}^{-1}$ & Erosion généralisée \\
\hline
\end{tabular}


Xèmes Journées Nationales Génie Côtier - Génie Civil, 14-16 octobre 2008, Sophia Antipolis

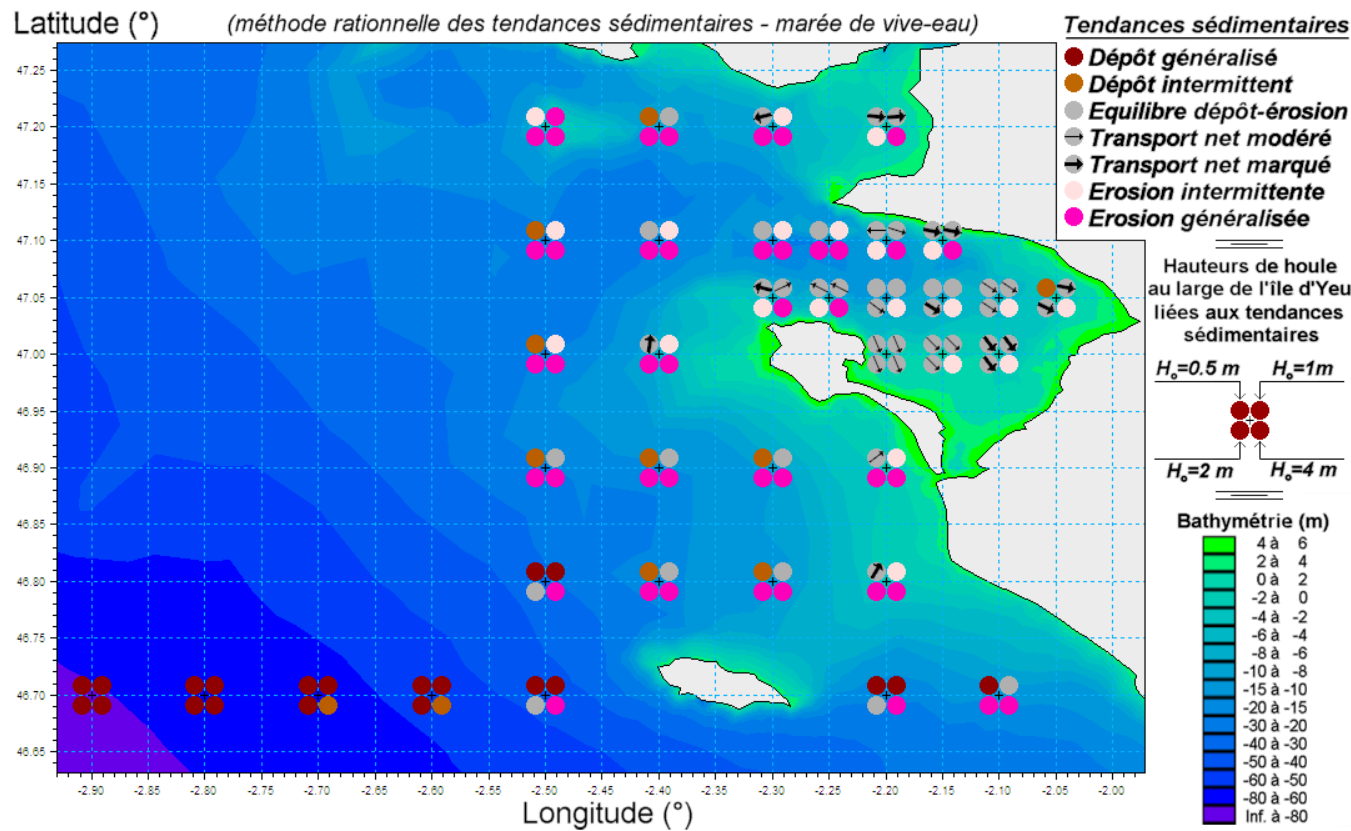

Figure 3. Tendances sédimentaires modélisées en 36 points du littoral atlantique proches de l'embouchure de la Loire, pour une marée de vive-eau moyenne et quatre conditions de houle caractérisées par la hauteur significative $H_{o}$ au large de l'île d'Yeu.

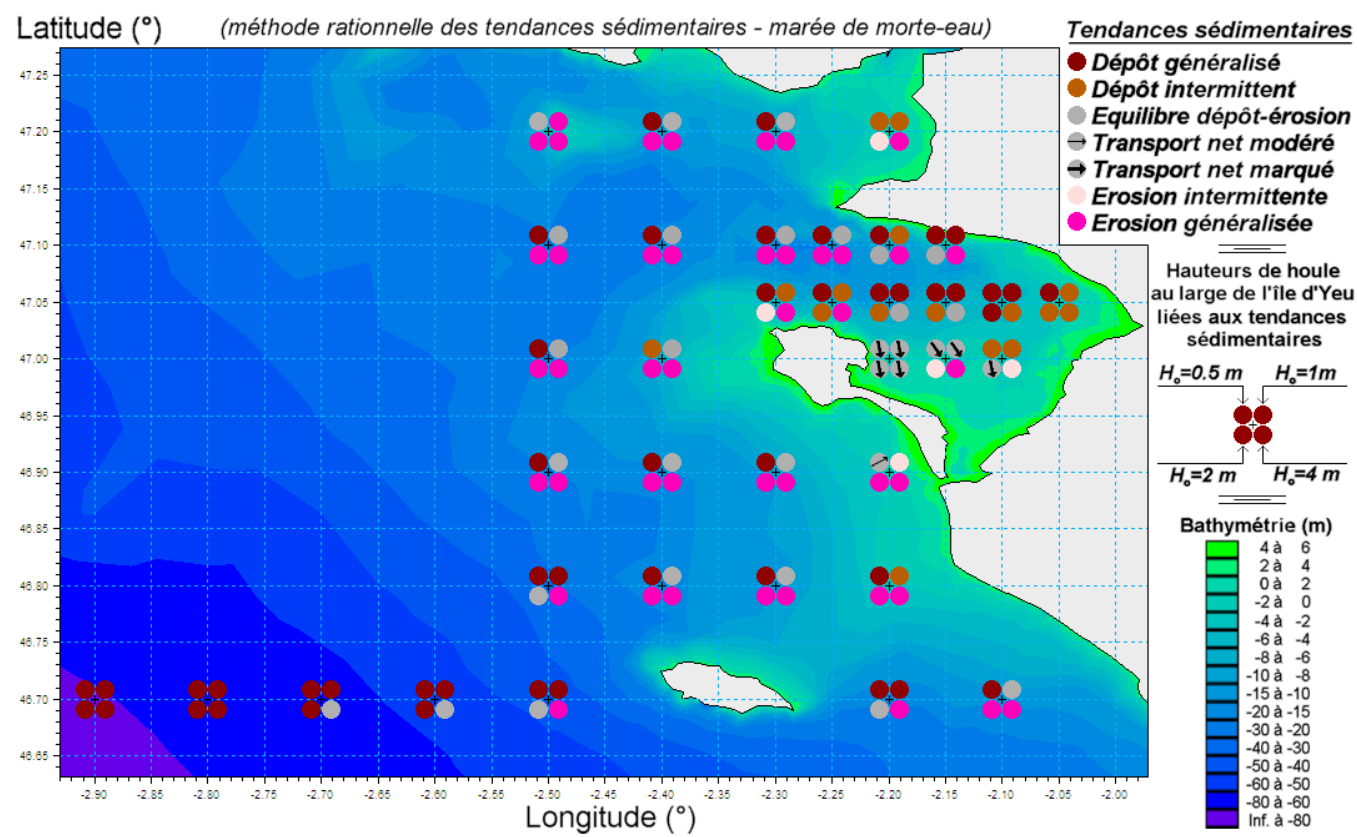

Figure 4. Tendances sédimentaires modélisées en 36 points du littoral atlantique proches de l'embouchure de la Loire, pour une marée de morte-eau moyenne et quatre conditions de houle caractérisées par la hauteur significative $H_{o}$ au large de l'île d'Yeu. 


\section{Discussion}

La signification de chacune des tendances sédimentaires mentionnées dans le tableau 1 et aux figures 3 et 4, est décrite par la suite.

\subsection{Tendance à un dépôt généralisé ou intermittent}

La tendance à un dépôt est liée à des contraintes hydrodynamiques près du fond faibles ne pouvant pas entraîner une remise en suspension significative de sédiments fins déposés. Si la tendance au dépôt est prédominante, les fonds peuvent alors s'engraisser de sédiments sur le très long terme. Il est vraisemblable que les turbidités resteront toujours très faibles, de type océaniques, bien inférieures à $0.005 \mathrm{~kg} \mathrm{~m}^{-3}$, et ainsi, le processus d'envasement sera extrêmement lent, imperceptible à une échelle de temps humaine. Le point le plus au sud-ouest du domaine océanique représenté sur les figures 3 et 4 est situé dans la Grande Vasière (figure 1) et correspond en effet à ce cas précis.

\subsection{Equilibre entre dépôt et érosion}

Les agents hydrodynamiques mettent en suspension les sédiments fins qui sont ensuite transportés en suspension suivant les courants de marée pour se déposer quand les eaux deviennent suffisamment calmes. Cependant, aucun déplacement résultant significatif n'est relevé $\left(v_{r} \approx 0\right)$ car le transport par le courant de flot est contrebalancé par celui provoqué par le courant de jusant. Un effet dispersif engendré par les courants de marée doit néanmoins être pris en compte tant que les sédiments restent en suspension.

\subsection{Tendance à un transport net des matières en suspension}

Les dissymétries de la marée qui sont dues principalement aux faibles profondeurs, expliquent dans certaines conditions de marée et de houle, une tendance à l'érosion à la basse mer et une tendance au dépôt à la pleine mer. Le flot transporte alors vers le rivage, plus de sédiments que ceux ramenées vers le large par le jusant, si bien qu'une vitesse résultante peut être évaluée pour les matières en suspension. Dans certains cas rares, le transport par le courant de jusant peut être prépondérant sur celui lié au courant de flot.

\subsection{Tendance à une érosion généralisée ou intermittente}

Cette tendance correspond à des contraintes hydrodynamiques près du fond élevées empêchant tout dépôt des matières en suspension. Les sédiments fins du fond sont alors susceptibles d'être mis en suspension. Si la tendance à l'érosion est prédominante, la présence des dépôts de vase ne peut être que sporadique et très éparpillée ; les turbidités restent ainsi faibles la plupart du temps. 
Une tendance à l'érosion se traduit par un maintien des sédiments en suspension qui dérivent lentement suivant les courants de marée résiduels. De plus, les courants de marée engendrent un important effet dispersif tendant à distribuer les sédiments depuis les zones de forte turbidité vers les zones peu turbides.

\section{Conclusions}

La distribution des dépôts marins de sédiments fins à proximité de l'embouchure de la Loire montrée sur la figure 1 peut s'expliquer avec cohérence par la méthode rationnelle des tendances sédimentaires développée dans cette note. Cette méthode permet d'expliquer également les processus dynamiques affectant les sédiments fins dans le domaine océanique étudié.

La forte accumulation des sédiments en baie de Bourgneuf s'explique en particulier par une érosion dominante à basse mer, quand les profondeurs sont faibles. L'érosion est suivie d'un transport vers le rivage par le courant de flot et d'un dépôt aux alentours de la pleine mer. Ce mécanisme qui engendre une vitesse résultante tendant à rassembler les matières en suspension au sud et à l'est de la baie s'oppose à la dispersion des sédiments vers les grandes profondeurs par les courants de marée. Il contribue ainsi au maintien d'une zone de forte turbidité sur le long terme.

Par opposition à la baie de Bourgneuf, la Grande Vasière située à une centaine de kilomètres des côtes de la façade atlantique (figure 1) est une zone de faible turbidité où les dépôts fossiles sont peu remaniés par les agents hydrodynamiques. Enfin, il doit être indiqué qu'aucun dépôt significatif de vase n'est répertorié là où des conditions d'érosion sont relevées en vive-eau (figures 1 et 3 ) pour une hauteur de houle moyenne $\left(H_{o}=2 \mathrm{~m}\right)$ ou supérieure à celle-ci.

\section{Remerciements}

Cette étude est liée au projet GERRICO piloté par l'IFREMER et l'Université de Nantes avec le soutien de la Région des Pays de la Loire. L'auteur remercie en particulier Hélène OGER-JEANNERET et Marc ROBIN, co-responsables scientifiques de l'axe 3 "interactions nature/société", ainsi que Benoist HITIER et Laurent BARILLE qui sont associés à ce même axe de recherche.

\section{Références bibliographiques}

${ }^{1}$ ALLEN G.P. (1972). Etude des processus sédimentaires dans l'estuaire de la. Gironde. Thèse, Université de Bordeaux, 314 p.

2 ALLEN G.P., CASTAING P. (1977). Carte de répartition des sédiments superficiels sur le plateau continental du Golfe de Gascogne. Bulletin de l'Institut de Géologie du Bassin d'Aquitaine, pp 255-260. 
${ }^{3}$ BARUSSEAU J.-P. (1973). Evolution du plateau continental rochelais (Golfe de Gascogne) au cours du Pléistocène terminal et de l'Holocène. Thèse, Université de Bordeaux I, $363 \mathrm{p}$.

${ }^{4}$ CANDHIS (site web). http://www.cetmef.equipement.gouv.fr/donnees/candhis/

${ }^{5}$ DELANOË Y., DIEUCHO A., GOULEAU D. (1971). Structures et formations sédimentaires de la baie de Bourgneuf (Loire-Atlantique) étudiées par sondage sismique réflexion. Comptes Rendus de l'Académie des Sciences, série D, n 272, pp 797-799.

${ }^{6}$ DHI (2007a). MIKE 21 \& MIKE 3 flow model FM. Hørsholm, Denmark, 14 p.

${ }^{7}$ DHI (2007b). MIKE 21 wave modelling. Hørsholm, Denmark, 16 p.

${ }^{8}$ FREDSØE J., DEIGAARD R. (1992). Mechanics of coastal sediment transport. World Scientific, Singapore, 369 p.

${ }^{9}$ FROIDEFOND J.-M., DOXARAN D., MILLER P. (2003). Rapport final : acquisition et traitement d'images satellites. Programme interrégional Loire grandeur nature, Cellule de mesures et de bilans Loire estuaire, Nantes, $174 \mathrm{p}$.

${ }^{10}$ GOULEAU D. (1975). Les premiers stades de la sédimentation sur les vasières littorales atlantiques - Rôle de l'émersion. Thèse d'Etat, Université de Nantes, $365 \mathrm{p}$.

${ }^{11}$ IPPEN A.T. (1966). Estuary and coastline hydrodynamics. McGraw-Hill, New York, $744 \mathrm{p}$.

12 KRONE R.B. (1986). The significance of aggregate properties to transport processes. In A.J. Mehta (Ed.), Estuarine cohesive sediment dynamics, Coastal and estuarine studies $n^{\circ} 14$ (pp 66-84). Springer, Berlin.

${ }^{13}$ LCHF (1987). Catalogue sédimentologique des côtes françaises - Côtes de la Manche et de l'Atlantique. Eyrolles, Paris, $559 \mathrm{p}$.

${ }^{14}$ LESUEUR P., JOUANNEAU J.-M., BOUST D., TASTET J.-P., WEBER O. (2001). Sediment rates and fluxes in the continental shelf mud fields in Bay of Biscay (France), Continental Shelf Research, n² 21 , pp 1383-1401.

${ }^{15}$ PARTHENIADES E. (1965). Erosion and deposition of cohesive soils. Journal of the Hydraulic Division, ${ }^{\circ}$ 91, pp 105-139.

16 SANCHEZ M., LEVACHER D. (2007). The influence of particle size of the dispersed mineral fraction on the settlement of marine and estuarine muds. GeoMarine Letters, ${ }^{\circ} 27$, pp 303-313.

${ }^{17}$ SHOM (1990). Courants de marée de la côte sud de Bretagne de Penmarc'h à Noirmoutier. Service hydrographique et océanographique de la marine, Paris, $48 \mathrm{p}$.

18 VANNEY J.-R. (1977). Géomorphologie de la marge continentale sudarmoricaine. Société d'édition d'enseignement supérieur, Paris, 473 p. 